\title{
EL PROYECTO BOTÁNICO DE LA EXPEDICIÓN MALASPINA
}

\section{Andrés Galera}

\section{RESUMEN}

Finalizando el siglo XVIII la monarquía de Carlos III patrocina la expedición Malaspina con el objetivo de explorar los continentes de América, Asia y Oceanía. En su apartado político el viaje perseguía la reforma del obsoleto modelo colonial español y en su faceta científica se desarrolló un proyecto de investigación acorde a los intereses de Europa. Nuestro objetivo es definir los estudios botánicos realizados por los naturalistas de la expedición Malaspina en relación con la politica científica que desde el Real Jardin Botánico de Madrid la corona españoia proyectaba para sus territorios de ultramar.

\section{SUMMARY}

Concluding the XVIIIth Century the monarchy of Carlos III patronizes the expedition Malaspina with the goal of exploring the continents of America, Asia and Oceania. On their political aspect the journey persecuted the reformation of the obsolescent colonial Spanish model, and in their scientific facet the developement of a project of investigation in agreement with the European interests. Our goal is define the botanical studies accomplished by the naturalists of the expedition Malaspina in relationship with the scientific policy that from the Royal Botanical Garden from Madrid the Spanish crown projected for their overseas territories.

Asclepio-Vol. XLVII-2-1995 


\section{ANDRÉS GALERA}

El continente bibliográfico y actos conmemorativos que en las últimas décadas de nuestro siglo han monopolizado la figura de Alessandro Malaspina y su Viaje científico y político alrededor del mundo, resarce con generosidad el ostracismo padecido por la expedición a su regreso en septiembre de 1794. Extremos peligrosos ambos. Bajo la sombra de tan frondoso árbol, hablar de botánica y hacerlo con el referente de la expedición Malaspina puede parecer un ejercicio reiterativo y nada novedoso. Cantar y contar las excelencias recolectoras de Louis Neé y Tadeo Haenke durante el viaje $^{1}$, y la posterior utilización de sus herbarios por naturalistas como Cavanilles, Lagasca, Candolle, y Presl para describir nuevos géneros y especies, en calidad de garantes de los trabajos botánicos malaspinianos, sería repetitivo y frustrante. Aplicando las enseñanzas del propio José Cavanilles, aquéllos tienen «la parte de la gloria que merecieron viajando y secando esqueletos», a éstos les corresponde «la que resulta del examen y trabajos científicos». Ciertamente, como afirma el naturalista valenciano, «no es lo mismo ser viajante que botánico; ni ver plantas y ser juez competente para determinar la fructificación, género y especie»². Cavanilles se interroga sobre el oficio de botánico, señala las diferencias entre quien circunscribe su labor al gabinete y quien la realiza inmerso en la naturaleza buscando sus secretos. La disputa se acentúa al legitimar el reconocimiento intelectual, al distribuir los honores correspondientes a uno y otro papel. Pero la cuestión merece un punto y aparte al que no podemos atender en esta ocasión. El ejemplo malaspiniano conlleva ambas facetas de la taxonomía vegetal, revela la categoría de Haenke, responsable de la octava edición del Genera Plantarum linneano ${ }^{3}$, que poco o nada debe demostrar, frente a un Louis Neé que a sus 54 años tiene todavía mucho camino por recorrer. Nos interesa, sin embargo, alejarnos de la individualidad y analizar el significado colectivo a las luces, pocas o muchas, de la comunidad botánica española durante los reinados del tercer y cuarto Carlos.

Un referente obligado en esta búsqueda de identidad es el Real Jardín Botánico matritense, institución donde Casimiro Gómez Ortega estableció

\footnotetext{
${ }^{1}$ Las cerca de 16.000 plantas recolectadas por Neé (véase la relación botánica elaborada a este respecto por Neé bajo la denominación de "Plantas colectadas en la expedición alrededor del Mundo», Archivo Museo Naval, doc. Exp. Malaspina, ms 1.407, f. 81), y los aproximadamente 15.000 ejemplares, fruto de la herborización de Haenke.

2 Cavanilles, A. J. (1796), Papeles sobre controversias botánicas, Madrid, Imp. Real introducción, p. 12.

${ }^{3}$ Linneo, C. (1791), Genera Plantarum, Wien.
} 


\section{EL PROYECTO BOTÁNICO DE LA EXPEDICIÓN MALASPINA}

sus reales sobre las directrices botánicas a seguir respecto a los recursos vegetales de ultramar.

\section{El jardin de Casimiro}

En 1803 el naturalista José Cornide calificaba el Real Jardín Botánico de Madrid como

«el plantel o semillero de tantos jóvenes como se han dedicado a la botánica, y que recorriendo los países más distantes le han enriquecido con numerosas y exquisitas plantas aún no conocidas en Europa» ${ }^{4}$.

El aserto testimonia la realidad de la institución matritense desde su fundación en 1755, como lugar de enseñanza botánica y centro de investigación sobre la flora americana. Ambos objetivos responden tanto a la finalidad docente con la que fue creado, como al exclusivo carácter utilitarista de la ciencia asumido por la Casa de Borbón durante la segunda mitad del siglo; diseño que tuvo en Casimiro Gómez Ortega, primer catedrático del Jardín durante el período 1771-1801, un significado baluarte. Pero hagamos un breve paréntesis. Al interpretar la naturaleza, Diderot establece dos categorías de filósofos:

"unos tienen, me parece, muchos instrumentos y pocas ideas; los otros tienen muchas ideas y no tienen ningún instrumento" ${ }^{5}$.

Se trata de la clásica entelequia baconiana entre experimentación y raciocinio, entre observación y reflexión, que aconseja equilibrar uno y otro bando. Junto a tal dicotomía, no parece desdeñable considerar en nuestro balance histórico una nueva clase de científicos: aquéllos que no tienen ni ideas ni instrumentos, pero sí prestigio; donde Gómez Ortega se mueve como pez en el agua. El boticario Casimiro ejemplariza el adagio sobre la valía científica en relación con la capacidad política del individuo y no por

\footnotetext{
${ }^{4}$ CoRnide, J. (1983), Ensayo de una descripción física de España, ed. H. Capel \& L. Urteaga, Barcelona, edicions Univ. de Barcelona, p. 62.

5 Diderot (1992), Pensées sur l'interprétation de la nature, ed. M. Jalón, Barcelona, Anthropos, p. 3.
} 


\section{ANDRÉS GALERA}

su intelecto. Así, desde su cualificada morada botánica, supo atender e hizo valer los predicados de ilustrados gobernantes como Campomanes, Floridablanca, Aranda y Gálvez. Veamos un ejemplo. En su manuscrito Reflexiones sobre el comercio español a Indias $^{6}$, redactado hacia 1762, Campomanes anticipa los ingredientes de la futura doctrina botánica orteguiana: la importancia de los conocimientos botánicos como factor económico, "conocimientos sin los cuales no se puede adelantar el comercio» ${ }^{7}$; consecuentemente, se pone el acento en «la utilidad que la historia natural y la botánica de España sacarían de hacer trasplantar los árboles y plantas referidas al Jardín Botánico del Rey y a los Jardines Reales» ${ }^{8}$. Política científica dirigida a explorar los territorios de ultramar, buscando un saber en aras del conocimiento, sí, pero más aún en beneficio del erario, mediante la comercialización de los recursos vegetales; modelo nada novedoso, a la zaga de avezados y diligentes competidores por el tesoro vegetal del Nuevo Mundo:

«es cierto que muchas plantas o árboles trasladados de un país a otro podrán perder mucho de su actividad o, acaso, adquirirla igual o mayor. Lo que será siempre seguro es que el conocimiento de estos vegetales se extenderá, y hará familiar a los Curiosos y Profesores que acudan al Jardín Botánico. Los holandeses hicieron a toda costa dibujar las plantas de su Colonia de Suriñán en la América. Fácil cosa es el convencer la mayor importancia de dar a conocer en España estos países mucho más fértiles y recomendables» ${ }^{9}$.

Mensaje que Ortega llevó a la práctica concibiendo el jardín botánico como una institución dedicada a una sistemática vegetal acorde con los parámetros linneanos, y a la propagación de plantas útiles ${ }^{10}$; cuyo brazo ejecutor fueron las expediciones botánicas. Siguiendo esta doctrina y los auspicios de la Corona, Hipólito Ruiz, José Pavón y el naturalista francés Dombey reconocen los territorios de Perú y Chile en el período 1771-1788; José Celestino Mutis dirige la Real Expedición Botánica al Nuevo Reino de Granada en

\footnotetext{
${ }^{6}$ Rodríguez Campomanes, P. (1988), Reflexiones sobre el comercio español a Indias, edición de Vicente Llombart, Madrid, Instituto de Ėstudios Fiscales, 1988.

7 Ibidem, p. 85.

8 Ibidem, p. 120.

9 Ibidem.

10 Véase GÓmEz ORTEGA, C. (1779), Instrucción sobre el modo más seguro y económico de transportar plantas vivas por mar y por tierra a los países más distantes, Madrid, J. Ibarra, pp. 9 y 10
} 


\section{EL PROYECTO BOTÁNICO DE LA EXPEDICIÓN MALASPINA}

el periplo 1783-1808; Martín Sessé, director de la Real Expedición Científica a Nueva España, sigue la huella de Francisco Hernández durante los años 1787 a 1803; y Juan Cuéllar explora las islas Filipinas de 1786 a 1797. En este contexto, ¿qué papel representa el reino vegetal en el horizonte de la expedición Malaspina? El proyecto malaspiniano trae aires de cambio. Ni la botánica lidera el universo cognitivo de la expedición, ni Casimiro Gómez Ortega dirige el quehacer de los naturalistas, ni el Real Jardín Botánico ostenta el protagonismo institucional en beneficio del Real Gabinete de Historia Natural, convertido en la enseña científica de la Corona. Veamos cómo.

\section{Otras áreas cognoscitivas}

«Dar una idea más completa del globo en general y del hombre que lo habita» ${ }^{11}$, es la máxima del viaje alrededor del mundo emprendido por las corbetas Descubierta y Atrevida en julio de 1789. Con Alessandro Malaspina nos alumbra la luz del modelo diderotiano pergeñado en el Suplemento al viaje de Bougainville, luz que, junto a la búsqueda de una mayor seguridad en la navegación y precisión cartográfica, compone un discurso náutico presente también en los bordos del marino italiano por los mares del Nuevo Mundo. Si «filosofía, coraje, veracidad; un golpe de vista rápido que capta las cosas y abrevia el tiempo de la observación; circunspección, paciencia, deseo de ver, de esclarecer e instruirse; la ciencia del cálculo, de la mecánica, geometría, astronomía y un barniz suficiente de historia natural» ${ }^{12}$, eran cualidades propias del navegante Bougainville en su circunvalación del globo, no faltan en Malaspina, y se multiplica el aporte científico gracias al desarrollo de la Europa ilustrada. Así, finalizando el siglo el estudio de la naturaleza, el barniz de historia natural, es un cuadro multicolor donde física, química, geología, zoología y botánica, colaboran en una investigación "que puede producir ventajas considerables a la monarquía, y a el progreso de las ciencias» ${ }^{13}$, sin olvidar la glo-

11 Estado general de los aprestos para la expedición, que de orden de S. M. ha de trabajar las costas de la América. Meridional, islas Marianas, y Filipinas», 27 de febrero, 1789. Archivo Museo Naval, doc. Exp. Malaspina, ms 583, f. 36v.

12 Diderot (1992), Suplemento al viaje de Bougainville, ed. de M. Jalón, Madrid, Debate/CSIC, p. 62.

${ }^{13}$ Carta de A. Malaspina a Tadeo Haenke comunicándole las instrucciones para su viaje hasta Buenos Aires, Lima 13 de septiembre 1793. Archivo Museo Naval, doc. Exp. Malaspina, ms 427, f. $96 \mathrm{v}$. 


\section{ANDRÉS GALERA}

ria particular del científico. Utilitarismo, universalidad y fama, son los argumentos de estos mercaderes de luz que construyen la ciencia tanto en la expedición Malaspina como en la utópica Sinapia legada por Campomanes, en un contínuo peregrinar "por todas partes adquiriendo libros, noticias, materiales y modelos para el adelantamiento de la ciencia y artes ${ }^{14}$; cimientos sobre los que descansa la fábula de la sociedad moderna.

«Todo lo que presente la superficie en los diferentes parajes que visitemos» ${ }^{15}$. En este infinito saber que define los intereses de la expedición Malaspina sobre historia natural, la botánica ocupa un lugar secundario. La flora representa un conocimiento tradicional en la exploración del nuevo mundo, herborizar es una práctica incluso rutinaria con el paso de los siglos, vinculada a esa presencia terrenal aludida por Alessandro Malaspina. Inventariar el reino vegetal es un hecho consustancial a cualquier viaje terrestre, un cometido recolector identificado por el herbario y la sistemática, que a finales del siglo XVIII, en el marco de un proyecto científico multidisciplinar, es superado por ámbitos más novedosos y controvertidos promovidos desde la Ilustración. Es el caso que nos ocupa. Además, en 1789 ni siquiera la ficción fisiocrática, el oro vegetal americano, puede ofrecer a la Corona la rentabilidad económica necesaria a sus dispendios. Ya no es suficiente andar "con ojos botánicos" los dominios de la monarquía, y las pesquisas oficiales dirigen su atención hacia la mineralogía, capaz de ofertar mayor e inmediata liquidez: "pues es el objeto único del viaje [recuerda Malaspina a Haenke para su tránsito por la cordillera andina], el aumentar los conocimientos de la historia natural en todos sus ramos, y facilitar los progresos del beneficio de minas» ${ }^{16}$. La monarquía acudía a la ciencia, no tanto para construir un modelo de sociedad sustentado en la proliferación de la cultura, como para remediar el desastre económico. La botánica poco o nada puede contribuir, pues la falta de una política agraria adecuada a las producciones naturales de la América espa-

14 AVILÉs, M. (ed.) (1976), Sinapia. Una utopía española del siglo de las Luces, Madrid, Editora Nacional, Biblioteca de Visionarios Heterodoxos y Marginados, p. 126. Si bien no se puede atribuir a Campomanes la autoría del manuscrito, este ha llegado a nuestros días gracias a su legado bibliográfico.

15 Carta de A. Malaspina a Antonio Pineda, Cádiz 26 de diciembre 1788. Archivo Museo Naval, doc. Exp. Malaspina, ms. 427, f. 3-4.

${ }^{16}$ Instrucciones de A. Malaspina a T. Haenke para su viaje por la cordillera de los Andes, realizado durante la estancia de la expedición en el pueblo de la Magdalena, Lima, Magdalena, 14 de abril 1790. A.M.N., doc. Exp. Malaspina, ms 426, f. 40v-41. 


\section{EL PROYECTO BOTÁNICO DE LA EXPEDICIÓN MALASPINA}

ñola, asegura su precariedad y la inviabilidad de cualquier experiencia agrícola, imposibilitando toda exigencia económica y demandando inversiones para su desarrollo. «Estas, sin embargo, serán siempre las resultas que deben esperarse de una dependencia directa del gobierno, en los asuntos mercantiles, pues por lo común no alcanzando a sus deseos la robustez del erario, y no siendo posible, sin una infracción de las leyes, el disponer arbitrariamente del caudal de un particular a favor de otro, últimamente desvanecen las promesas, y la sola falta del techo causa la ruina de todo el edificio» " ${ }^{17}$, afirma Malaspina valorando la precaria situación del plantío de canela de la hacienda de Calaván, en Manila.

\section{Nombres e instituciones}

En 1791, escribía Gómez Ortega al ministro de marina Valdés comunicándole que «tendré que trabajar coadyuvando a la coordinación y corrección de los manuscritos de mis discípulos Don Antonio Pineda y Don Luis Neé, empleados por V. E. en calidad de botánicos y naturalistas en el viaje más útil y glorioso que se haya hecho alrededor del mundo» ${ }^{18}$. Los discípulos ya se habían emancipado y la anunciada colaboración era imaginaria. Mal podía coordinar Casimiro los trabajos zoológicos de Antonio Pineda, y difícilmente Louis Neé aceptaría la autoridad del maestro que le había postergado. La falsedad es sintomática del declinar político y científico de Ortega. Déficit presente ya en la fase organizativa de la expedición, con significado tanto en la persona del catedrático como en el jardín botánico regentado. Su participación en el proyecto científico malaspiniano es puramente consultiva. La ausencia de otro norte botánico que la realización de herbarios otorga a la disciplina un carácter meramente recolector, viendo desplazado su liderazgo científico en favor de la zoología; materia fuente de una novedosa investigación sobre anatomía, fisiología y sistemática animal; amplitud de miras que contrasta con la linde taxonómica propia del reino vegetal. El final de la aventura confirma el

\footnotetext{
17 Carta de A. Malaspina a J. Cuellar. Manila 12 de septiembre 1792. A.M.N., doc. Exp. Malaspina, ms 427, f. 183.

${ }^{18}$ Carta de C. G. Ortega a Valdés, 1 de marzo 1791. Archivo General de la Marina, El Viso del Marqués, sec. Indiferente. Jardín Botánico. Cit. en GALÁN DE AHumadA, La farmacia y la botánica en el Hospital Real de Marina de Cádiz, Granada, tesis doctoral inédita, pp. 338-340. También en Puerto, F. J. (1992), Ciencia de cámara. Casimiro Gómez Ortega (1741-1818) el científico cortesano. Madrid, CSIC, p. 227.
} 
declive orteguiano. Habiendo fallecido en el transcurso del viaje Antonio Pineda, responsable de los estudios zoológicos, la organización de sus trabajos se encomendó a José Cornide; Neé rescató sus herbarios con la intención de publicar la flora de las, aproximadamente, 16.000 plantas recolectadas. La participación de Ortega quedó reducida a elaborar un informe favorable a la contratación del pintor José Guío como encargado de las láminas botánicas de una obra que no vio la luz.

Simultáneamente la expedición muestra el valor en alza del Gabinete frente al Jardín Botánico. Si en 1776 la instrucción elaborada por Gómez Ortega para la excursión botánica de Ruiz y Pavón por la América meridional, ya contemplaba el Real Gabinete de Historia Natural como el Gabinete del rey, escaparate de las riquezas animales, minerales y vegetales existentes en sus dominios, y por consiguiente las colecciones «de semillas, y frutos secos, de gomas, resinas, bálsamos, y demás productos o partes de las plantas, que tuvieren algún uso, o merecieren tenerle»19, tienen cabida en dicha institución, una década más tarde el hecho es una realidad, y el objetivo de la exploración malaspiniana «será el acopio de las cosas nuevas para el Real Gabinete de Historia Natural», dejando sólo el envío «de plantas vivas para el Real Jardín Botánico" ${ }^{20}$. Se definen dos niveles botánicos diferenciados, el coleccionismo concebido para el Gabinete, y el cultivo de plantas en manos del Jardín. ¿Cuál es la causa de este reparto de funciones? La expedición Malaspina es una aventura científica diferente a las empresas promovidas por la monarquía en ocasiones precedentes. Situada en el marco de los viajes protagonizados por Bougainville, Cook y La Pérouse, su ciencia gira sobre dos premisas: la grandiosidad de la Corona y la universalidad del saber. Los nuevos conocimientos contribuyen al progreso de la ciencia europea, y el empirismo, los objetos recolectados, manifiestan el poder de la Corona. En consecuencia, el patriotismo maląspiniano convierte al Gabinete en la institución que debe acoger el patrimonio nacional. «Y el gabinete es el libro donde lees / Quien

\footnotetext{
19 Instrucción a que deberán arreglarse los sujetos destinados por S.M. para pasar a la América meridional en compañía del médico Josef Dombey, a fin de reconocer las plantas, y yerbas y de hacer observaciones botánicas en aquellos países. Reproducido en RuIz, H. (1952). Relación del viaje que hizo a los reinos del Perú y Chile el botánico D. Hipólito Ruiz en el año de 1777 hasta el de 1788, en cuya época regresó a Madrid, ed. J. Jaramillo Arango, Madrid, p. 400.

${ }^{20}$ Carta de Malaspina a Antonio Pineda. Cádiz 26 de diciembre 1788. A.M.N., doc. Exp. Malaspina, ms. 427, f. 3v.
} 
eres y lo mucho que posees», explican los versos del poeta Iriarte ${ }^{21}$. El antecedente protagonismo botánico pasa a formar parte de una quimera nacional, «único juez a quien debemos tributar nuestros esfuerzos» afirma Malaspina ${ }^{22}$, cuyo interlocutor no es ya la institución que estudia los herbarios, sino quien los exhibe: «Los ejemplares secos de que se sacasen los dibujos serán conservados y colocados con los demás en el herbario que se debe formar para el Real Gabinete, como monumento eterno de un viaje que es el primero que se ha hecho por la Corte de España", afirmaba Neé a Cosme Churruca al exponer el plan de publicación de sus trabajos botánicos durante la expedición ${ }^{23}$.

En 1805 escribiría Humboldt a Bompland, respecto a la relación de su viaje, sobre el halago fácil y necesario con los botánicos españoles:

«haga una lista de las gentes que hay que alabar perpetuamente, y alabe a la vez a Neé, Zea, Mutis, Cavanilles, Sessé, Pavón y Ruiz y Tafalla y Olmedo. Yo he actuado así en mis manuscritos y es preciso que los suyos estén acordes con los míos, ya que formamos un solo equipo y quiero que se sepa que no tomamos ningún partido. Le conjuro a responder a Pavón lleno de amistad, sería desagradable tener problemas con ellos y podemos evitarlo» ${ }^{24}$.

Las facciones aludidas estaban representadas por Casimiro Gómez Ortega y José Cavanilles, y quien no pudo evitar los problemas fue Louis Neé. El botánico francés hacía tiempo que había respondido a Ruiz y Pavón, con bastante menos cordialidad de la reclamada por Humboldt. La solicitud por los botánicos de la expedición al Perú y Chile de los materiales recolectados por Neé, al objeto de incorporarlos a su flora Peruviana et Chilensis, originó la polémica:

${ }^{21}$ Epístola escrita por T. Iriarte a J. Cadalso, donde se describe en forma de poema la Academia de las Tres Nobles Artes y el Real Gabinete de Historia Natural; 28 de noviembre de 1776. Reproducida en BarreIro, A. (1992), El Museo Nacional de Ciencias Naturales (1771-1935); Edición de P.M. Sánchez, Madrid, Doce Calles, p. 361.

${ }^{22}$ Carta de Malaspina a Antonio Pineda, 28 de mayo 1792, corbeta Descubierta, A.M.N., doc. Exp. Malaspina, ms 427, f. 187.

${ }^{23}$ Informe de Louis Neé a Cosme Churruca sobre el plan de publicación de los trabajos botánicos. Madrid, marzo, 1796. A.M.N., doc. Exp. Malaspina, fol. 246v.

${ }^{24}$ Carta de Humboldt a Bonpland, Roma 10 de junio de 1805. En Humboldo, A. (1980), Cartas americanas, edición de Ch. Minguet, Biblioteca Ayacucho, Venezuela, pp. 149-52. 


\section{ANDRÉS GALERA}

«tardaron en su viaje once años, han tomado los agregados que juzgaron necesarios para ayudarlos, han tenido todos los auxilios necesarios y estuvieron todo el tiempo que quisieron estar en cada país. Creo que su colección ha sido de 6.000 plantas, ¿si en el tiempo de once años, entre tantas personas, han colectado sólo este número digo yo que no deben haber sudado mucho? 25 .

Fue la airada respuesta a tan arrogante petición. La cuestión de fondo es el disfrute de una heredad que se veía seriamente amenazada con la intromisión de Ruiz y Pavón. "Sería desacreditarme, quitarme mi hacienda ${ }^{26}$, explica Neé, renunciar no tanto a la gloria botánica como al beneficio económico que representaba su herbario; caudales con los que asegurar su vejez. Fue éste un episodio más del enfrentamiento entre Ortega y Cavanilles, característico de una disciplina y de una institución, el Jardín Botánico, que si no tiene estímulos cognoscitivos es la tribuna idónea para la proyección personal. Polémica que la expedición Malaspina significa en la figura de Neé, quien formó en las filas de Cavanilles.

La expedición Malaspina testimonia un fracaso botánico. Revés así en la explotación de recursos naturales, como en la evolución de la disciplina hacia temas de investigación impropios de la descripción y clasificación. Una tendencia propia de una ciencia botánica regida por el utilitarismo farmacológico, nacido de considerar al reino vegetal como mero instrumento médico. Interrogado en 1790 por el estado de la botánica española respondía Cavanilles:

«Poco menos que nada. Si Vm. exceptúa a Palau, que está casi decrépito e inútil ya para trabajar, todos los demás son poco menos que aprendices ${ }^{27}$.

Considerar aprendices a los botánicos españoles en el umbral de la centuria decimonónica resulta excesivo, pero no lo sería definirlos como nominalistas sin otro rumbo que la taxonomía.

${ }^{25}$ Carta de Luis Neé a Cosme Churruca, Madrid, marzo de 1796. A.M.N., doc. Exp. Malaspina, ms. 2296, fols. 244-6.

${ }^{26}$ Ibidem.

27 Carta de Cavanilles a J. Clavijo. Madrid 22 de septiembre 1790. En Cavanilles, J. (1981), Cartas a José Viera y Clavijo, introducción y notas A. Cioranescu, Tenerife, Aula de Cultura, p. 45. 